\title{
Téoros
}

Revue de recherche en tourisme

\section{Le tourisme sexuel en Thaïlande}

\section{Une prostitution entre misère et mondialisation}

\section{Franck Michel}

Volume 22, numéro 1, printemps 2003

Tourisme et sexualité

URI : https://id.erudit.org/iderudit/1071581ar

DOI : https://doi.org/10.7202/1071581ar

Aller au sommaire du numéro

Éditeur(s)

Université du Québec à Montréal

ISSN

0712-8657 (imprimé)

1923-2705 (numérique)

Découvrir la revue

\section{Citer cet article}

Michel, F. (2003). Le tourisme sexuel en Thaïlande : une prostitution entre misère et mondialisation. Téoros, 22(1), 22-28.

https://doi.org/10.7202/1071581ar d'utilisation que vous pouvez consulter en ligne.

https://apropos.erudit.org/fr/usagers/politique-dutilisation/ 


\section{Le tourisme sexuel en Thailande} Une prostitution entre misère et mondialisation

\section{Franck Michel}

\begin{abstract}
"Le sexe et l'esclavage sont des partenaires naturels dans un monde forgé par l'homme. En Asie, ils sont absolument inséparables. Les femmes esclaves sexuelles sont un produit intrinsèque de la domination masculine dans les sociétés asiatiques. Ils participent à un jeu vicieux que les hommes jouent avec les femmes. Elles sont les abusées, les stigmatisées, et le poids amer des valeurs asiatiques. Et elles ne peuvent pas, toujours, continuer à vivre et à mourir en silence »
\end{abstract}

Brown, $2000: 255$.

D rès de neuf millions de touristes ont visité la Thaïlande en l'an 2000, parmi lesquels 65 à $70 \%$ sont des hommes. Malgré d'intenses campagnes médiatiques de sensibilisation contre le tourisme sexuel et nombre de batailles judiciaires à l'encontre des abuseurs d'enfants en Asie et ailleurs, le secteur - si prospère pour beaucoup - du tourisme sexuel en Thaillande ne paraît guère vouloir se tarir. Au contraire, la demande s'élargit et se diversifie : après les « traditionnels » Occidentaux - ou plutôt en même temps qu'eux -, des Chinois fortunés, à l'instar des comités d'entreprises japonais, louent des autocars entiers de jeunes filles ; un tour opérateur israélien spécialisé dans les sex-tours d'un genre nouveau envoie exclusivement des femmes en mal d'affection quêter du fruit défendu auprès de prostitués à Bangkok ; les pédophiles continuent par ailleurs d'affluer à Pattaya, à Chiang Mai, à Bangkok ou ailleurs, et n'oublions surtout pas la masse impressionnante de « consommateurs » nationaux...
Certes, il y a vingt ans, Georges Cazes soulignait déjà :

Le tourisme international, s'il n'est pas à l'origine des salons de massage et des maisons de prostitution, leur a donné une sorte de consécration et de généralisation commerciale : il a contribué à aggraver l'émigration de la misère pendant la morte saison agricole des zones montagnardes $d u$ Nord et surtout des plateaux secs $d u$ Nord-Est, vers les agglomérations urbaines et balnéaires (1983 : 65).

Depuis, de trop nombreux tour opérateurs, peu soucieux d'éthique et convaincus que le sexe rapporte d'abord de l'argent, n'éprouvent guère d'états d'âme, comme le rappelle Bernard Formoso : "Le Farang qui visite dans la journée les palais et pagodes dorées et qui assiste le soir à un sex show ou s'essaye au 'massage' thaïlandais, entouré d'hôtesses en tenues légères, est un cliché d'une triste banalité » (2001: 57). Bref, rien donc de vraiment nouveau sous le ciel de Thailande, si ce n'est la perpétuation de ce « tourisme de la honte » (Michel, 2002 : 214-217) sous des formes réadaptées aux exigences d'une mondialisation effrénée. Le présent article dresse un rapide bilan du tourisme sexuel en Thaillande tout en tentant de déceler les racines du mal et d'explorer les moyens d'en éradiquer ses aspects les plus détestables, aux conséquences particulièrement morbides.

\section{Un bref état des lieux}

La Thailande, oasis de paix - devenue toute relative, notamment la zone de Phuket, depuis l'attentat de Bali en octobre 2002, et la volonté affirmée d'Al-Qaïda d'atteindre des « cibles molles » en Asie du Sud-Est -, au cœur d'un continent actuellement voué à l'instabilité chronique et aux soubresauts indéniables, bénéficie d'atouts certains qui expliquent en partie son «succès » dans ce secteur de l'économie, en dépit de sa face sombre qu'est l'industrie du sexe et de l'inquiétante proportion que celle-ci occupe au sein même de l'économie nationale. Mais que valent aujourd'hui des recommandations éthiques face à l'accumulation de chiffres tous plus prometteurs les uns que les autres:

À la fin des années 1990, près de 600000 emplois relevaient directement du tourisme et la Thaillande était de loin la première destination récréative en Asie du Sud-Est, ainsi que le principal lieu d'implantation des grandes chaînes hôtelières internationales dans la région. [...] Entre 1993 et 1998, le nombre de visiteurs est passé de 5,7 à 7,8 millions, soit d'une croissance d'un tiers en l'espace de cinq ans. Parmi ces touristes, $57,9 \%$ sont asiatiques, $26,8 \%$ sont européens, 6,8\% sont américains et 1,8\% proviennent du Moyen-Orient (Formoso, 2000 : 152-153).

La manne financière qui en découle est très inégalement répartie et l'activité touristique se concentre selon un axe nord-sud ayant Bangkok pour centre : "Le pôle de pauvreté du Nord-Est, quoique riche en sites archéologiques, était complètement tenu à l'écart des circuits des voyagistes et était de ce fait non équipé » (Ibid: 154). Sur ce « succès » repose également le terreau du tourisme sexuel qui, à ce jour, rapporte trop d'argent à tout le monde (l'État et les par- 
ticuliers) pour que des voix dissonantes puissent se faire entendre dignement.

Où en est-on aujourd'hui ? Loin des exagérations récurrentes, et citant notamment les travaux de Boonchalaksi et Guest (1994), Bernard Formoso avance le chiffre total de prostituées :

\section{[...] de 200 à 300 000, soit tout de même de 8,3 à 12,5\% des femmes de la tranche des 15-29 ans résidant en ville; auxquelles s'ajouteraient de 25000 à 30000 filles de moins de 15 ans et de 30 à 50000 garçons ou jeunes hommes qui satisfont la clientèle pédophile ou homosexuel- le. Si l'on s'en tient à cette hypothèse basse, il y aurait au milieu des an- nées 1990 de 250 à 380000 tra- vailleurs du sexe opérant en même temps (Formoso, 2001 : 58).}

Selon d'autres estimations, plus de 200000 enfants seraient aujourd'hui exploités dans l'industrie du sexe en Thaïlande. À Pattaya, par exemple, un policier local affirme que le nombre d'étrangers venant acheter du sexe était en augmentation de 1995 à 2000 et encore plus nettement depuis la crise de 1997 ; avant d'ajouter qu' ' il y a davantage d'enfants qui veulent se vendre eux-mêmes, c'est donc plus facile pour les pédophiles » (Dernières Nouvelles d'Alsace, 20 octobre 2000). On assiste à une sordide course de vitesse où, d'un côté, on voit une certaine prise de conscience du fléau et surtout un renforcement de la lutte contre le tourisme sexuel en particulier quand il concerne des enfants prostitués et, de l'autre, on observe avec une inquiétante impuissance l'explosion du marché de la chair et la nette augmentation de la demande...

\section{Clients étrangers... et locaux}

Sans revenir ici sur l'évolution amplement analysée de la prostitution en Thailande et sur l'inventaire de ceux qui s'y sont adonnés au fil de l'histoire - des coolies chinois du XIX ${ }^{\mathrm{e}}$ siècle jusqu'aux $\mathrm{GI}^{1}$ avec le programme Rest \& Recreation pendant la guerre du Vietnam, en attendant la relève des soldats perdus par des touristes « inoffensifs » en goguette -, on remarque que,

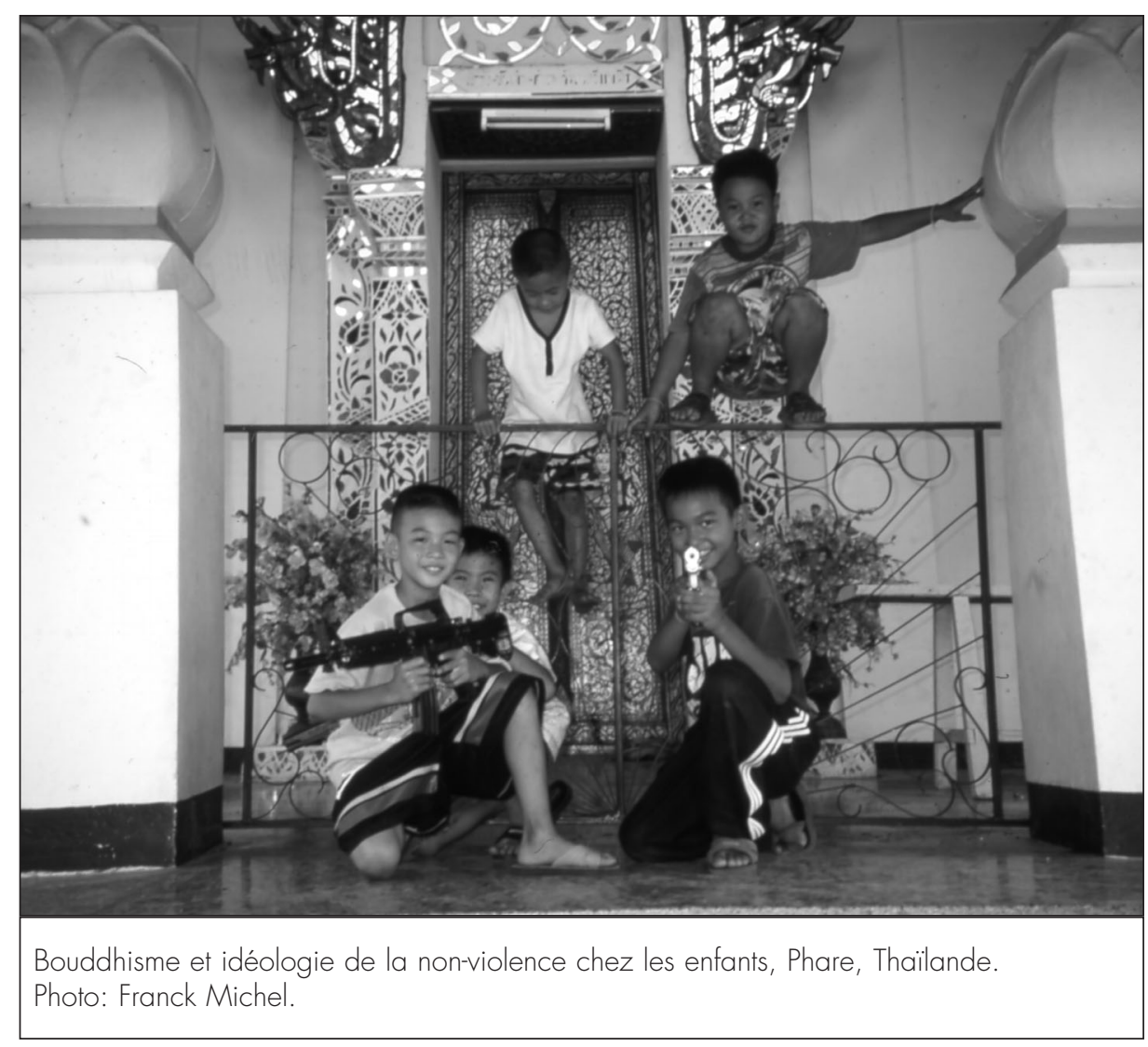

parmi les étrangers, les Occidentaux ont notamment développé une mythologie incroyablement féconde autour des vertus inestimables de la femme thailandaise. Films et romans ont largement alimenté et véhiculé ces images éculées d'impossibles icônes tant désirés et si désirables.

L'image de la femme thailandaise, avec son arsenal de confortables clichés pour Occidentaux en mal de virilité, est aussi utilisée sans scrupules par les prestataires et les organismes officiels de voyage : « Chiang Mai ne fascine pas seulement par la beauté de ses paysages, les femmes de la ville sont aussi considérées comme étant les plus belles de tout le pays et sont particulièrement fières », peut-on ainsi lire dans Reisen in Thailand (Bangkok, n5, mai 1994). Une autre brochure officielle vante et vend les qualités des Thailandaises comme suit : «Les femmes thaïlandaises sont reconnues pour leur grâce, leur gentillesse, leur politesse et leur nature attentionnée. Elles sont aussi des mères affectueuses et des femmes au foyer méticuleuses qui font d'elles de parfaites compagnes de vie » (What's on Where to Go, Thailand this Week, "Let Thai Contacts Be Your Cupid », Bangkok, 15 mai 1994). Une image fantasmée qui se transpose aussitôt sur le plan sexuel : dans l'imaginaire occidental, nourri de sentiment de supériorité et d'idéologie coloniale, les femmes thailandaises - et au-delà « asiatiques » - seraient donc faciles à conquérir, voire à posséder (Michel, 1995 et 1998).

De brochures quadri en cartes postales, puis de «tribaux » en tripots, les touristes découvrent de bien tristes tropiques ; Bernard Formoso déplore le fait que les visiteurs en Thaillande se contentent des habituels clichés et des itinéraires sans surprises :

\section{[...] ils ne retiennent que les rares sites «typiques » encore préservés dans la jungle urbaine de Bangkok (la cité royale, les grands temples, le marché flottant) ; auxquels s'ajou- tent Pat Pong, ses boîtes de nuit et son armée de filles de joie, les " tri- baux » du Nord mis en scène par les tour operators ou encore les plages de sable fin de Phuket et de Ko Samui (Formoso, 2000 : 154).}




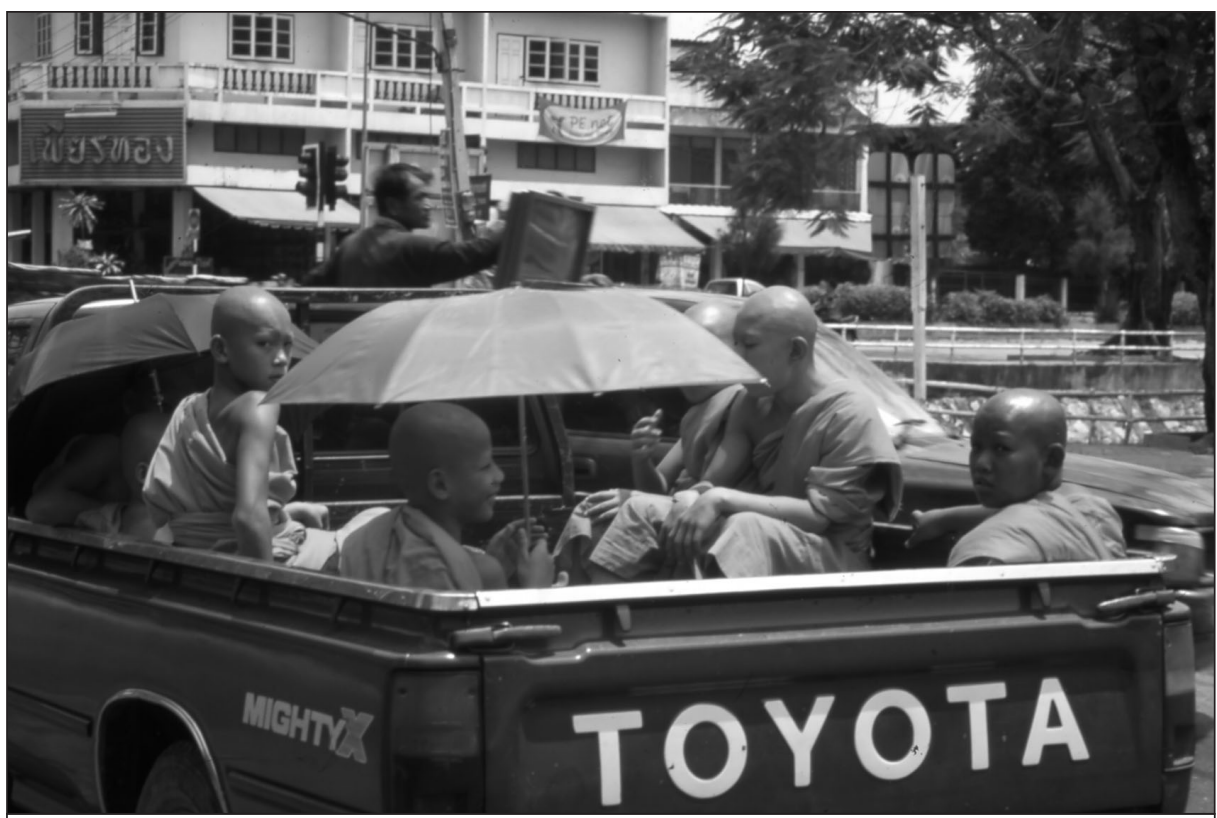

La modernité religieuse Thaïe : Bonzes et Toyota à Lampang, Thaillande. Photo: Franck Michel.

Empli, sinon conquis, par ces visions idylliques d'un pays tout en sensualité, le passage à l'acte est tentant pour le touriste en vacances, ce badaud anonyme loin de chez lui et de ses habituelles restrictions... Le profil des clients occidentaux est connu, souvent étudié en même temps que celui des prostituées, et varie accessoirement selon les lieux et les époques (Truong, 1990 ; Michel, 1995 ; Bishop, Robinson, 1998 ; Seabrook, 2001).

En plus des clients étrangers sur la sellette, depuis belle lurette - et pour cause -, il y a les clients «nationaux » bien trop oubliés, en partie en raison de l'aveuglement dû à la mauvaise conscience occidentale... Gardons en effet à l'esprit qu'aux yeux de la clientèle totale, les étrangers ne représentent qu'une faible proportion, ce que le livre de Louise Brown, Sex Slaves, s'attache à démontrer, car il s'agit dans ce domaine de récuser quelques idées reçues bien ancrées : l'industrie du sexe en Asie proviendrait essentiellement de la demande de touristes sexuels occidentaux... C'est ce que nous montrent sans arrêt les médias, il est vrai, notamment sur les exemples philippin et thaillandais. Si ces cas sont bien entendu évidents et même si le tourisme sexuel, tout comme la pédophilie, ne cessent aujour- d'hui de progresser dans la région d'une manière absolument dramatique, Brown rapporte à juste titre que la majorité des clients de prostitués femmes ou enfants sont avant tout des hommes asiatiques. Cela n'absout en rien évidemment les abuseurs occidentaux des enfants et des filles asiatiques, mais cela permet de rétablir une vérité tragique, bien loin de la gestion de notre culpabilité judéo-chrétienne caractéristique du débat en Occident. Et Louise Brown a le mérite de démolir des pans entiers de ce qui est à la base des trop fameuses «valeurs asiatiques », tout en montrant aussi que l'industrie du sexe est essentiellement le résultat d'une société intensément dominée par les hommes (Brown, 2000).

Une interprétation confirmée, d'une autre manière, par Bernard Formoso qui rappelle utilement que, dans un pays de « longue tradition de polygamie et de mercantilisation des femmes, avec une interprétation très accommodante de l'acte méritoire bouddhique et du fait aussi d'une économie nationale qui repose sur le principe des migrations tournantes, la demande intérieure prévaut ». Et l'ethnologue de souligner avec justesse que «La prostitution répond d'abord et avant tout aux désirs des hommes thaillandais » (Formoso, 2001 : 67).

\section{Prostituées locales... et étrangères}

En Thaillande, les prostituées sont jeunes et le plus souvent issues des zones rurales défavorisées du Nord et, dans une moindre mesure, du Nord-Est. Avec les ravages du sida, une prostitution plus clean a vu le jour, avec des « travailleuses » qui ont un niveau d'éducation supérieur et qui proviennent d'un milieu urbain. Le chercheur thailandais Phongpaichit a démontré que ces employées ou ces étudiantes qui s'adonnent à la prostitution sont d'abord soucieuses d'améliorer un quotidien «moyen » et de consommer un maximum selon les préceptes de l'idéologie officielle et libérale en vigueur dans le Royaume : les prostituées peuvent ainsi espérer gagner de 180 à près de 1000 dollars américains par mois, soit de deux à huit fois plus que ce qu'elles gagneraient si elles faisaient un autre travail (Phongpaichit, 1998 : 200-208).

En raison de l'ouverture des frontières, de la précarité des ménages, de la guerre et de la pauvreté des voisins, un nombre croissant de filles étrangères s' " installe » dans l'industrie du sexe thaïlandais. Aux côtés des Cambodgiennes, des Laotiennes et des Vietnamiennes, ce sont maintenant aussi les jeunes filles birmanes (notamment des minorités Tai Yai et Mon) et yunnanaises (du Sud-Ouest de la Chine) qui viennent chercher « refuge » ou « fortune » au pays du sourire éternel... Celles-ci, âgées de 12 à 18 ans, sont très demandées depuis que certaines Thaillandaises du Nord ont été persuadées - épidémie du sida aidant - de choisir d'autres voies. Ces esclaves sexuelles, immigrées clandestines par ailleurs, représentent un inépuisable vivier pour le tourisme sexuel des enfants, sans oublier qu'ici comme ailleurs «les travaux sexuels sont les mieux payés pour les enfants « 6281 bahts par mois [environ 1300 FF]); les enfants entrent en Thailande pour des raisons économiques, attirés par le désir de consommation et la corruption des autorités thaïlandaises »(Poona, 1997 : 4). Le tourisme sexuel, s'inscrivant également dans le contexte des nouvelles mobilités contemporaines suscitées par la mondialisation, s'exporte et se transporte au gré de la demande : d'un côté, des femmes blanches, blondes de préférence, prove- 
nant d'Europe orientale ou de Russie, répondent aux exigences d'une prostitution de luxe à destination des hommes d'affaires asiatiques ; de l'autre, des femmes thaillandaises sont toujours plus nombreuses à se prostituer au Japon, laissant ainsi le «bas œuvre » des bordels bon marché locaux aux Birmanes et aux Chinoises en quête de survie, de nourriture et de papiers.

Dans Sex Slaves, fruit d'une enquête sur l'exploitation sexuelle et le trafic des femmes dans tout le continent asiatique, Louise Brown dénonce fortement ce commerce de la honte, sur fond de tourisme sexuel et de corruption latente, en donnant la parole aux femmes les plus silencieuses et les plus abusées du monde. Leurs témoignages, à l'heure d'une mondialisation aux conséquences incontrôlables, révèlent l'univers sordide des jeunes filles enlevées ou vendues dans leurs villages reculés, la vie dans les bordels, les coups des mamasan, l'hypocrisie des clients, les ravages du sida et, toujours, le sort des femmes humiliées et laissées à elles-mêmes, dans le désespoir souvent le plus total (Brown, 2000). Les filles sont effectivement exploitées, parfois vendues sous contrat par leur famille, quelquefois même enlevées et forcées au travail dans des conditions d'esclavage.

Rappelons ici qu'une certaine forme de prostitution forcée, héritée de l'esclavage pour dette du $\mathrm{XIX}^{\mathrm{e}}$ siècle, perdure ici ou là en Thaillande, dans les poches de pauvreté extrême. Vendues ou "mises en gage », ces filles sont d'authentiques esclaves sexuelles, parfois sans le savoir et sans que leurs parents - qui les ont vendues délibérément - ne les perçoivent comme telles... La situation de maintes régions, plus vulnérables car plus misérables, en devient parfois tout simplement catastrophique : «Dans certains villages, la quasitotalité des filles en fin de scolarité obligatoire, et âgées de douze à quinze ans, deviennent des prostituées », rapporte Mayuree Rattanawannatip pour le compte du quotidien The Nation (29 juin 1990). La pauvreté reste sans doute la plus grande source de vocations, comme le laisse également entendre la journaliste du Bangkok Post, Sanitsuda Ekachai : «La prostitution est une activité banale parmi les jeunes femmes de certaines régions $d u$ Nord. Elle est devenue une source de revenus majeure et la 'clé de la survie' de l'ère moderne » (Sanitsuda, 1993 : 128).

Cela dit, il convient de noter la forte variabilité de l'univers de la prostitution en Thaillande : en effet, rien ou presque - à l'exception de l'oppression masculine dans la société - ne semble rapprocher l'étudiante qui se prostitue occasionnellement au cœur de Bangkok pour assouvir son envie de posséder le portable dernier cri et la fille abandonnée de tous, contrainte à se prostituer dans le seul but de survivre dans un bordel sordide aux confins birmano-thaillandais... De la même manière, on observe une importante distinction entre celles qui s'en sortent - par le mariage, notamment avec un Farang, ou grâce à une réinsertion aisée et réussie par exemple lors d'une « réinstallation » dans le village d'origine - et celles qui sombrent sans espoir de retour, par la maladie (sida, drogues...) ou encore sous l'effet des multiples formes de désespoir et d'autodestruction, si fréquentes dans le milieu glauque de la prostitution. En tant que puissant vecteur de la mondialisation, le tourisme génère son lot de représentations de l'Autre et de l'hôte et il n'échappe en rien à cette altérité en mouvement :

\section{La prostitution en Thaïlande procè- de toujours d'un rapport à "l'é-} tranger». Celui-ci peut être du pro- che ou du lointain, mais il vient d'ailleurs, à l'aune d'une pratique inscrite dans la mobilité. Le tourisme entre dans ce schéma, tout comme le Farang endosse involontairement les habits $d u$ "riche » polygame (Formoso, 2001 : 67).

\section{Trois " C ", trois fléaux : consumérisme, capitalisme, corruption}

Le drame aujourd'hui est simple : si la lutte contre le tourisme sexuel s'intensifie effectivement, le fléau semble se répandre et même se développer à une échelle beaucoup plus rapide. Sans compter que l'industrie du sexe rapporte à la Thaillande cinq à six fois plus que les recettes de la drogue, soit de 18 à 21 milliards de dollars américains en 1996. Dans de telles conditions, comment croire que les 60000 bordels que compterait le royaume pourraient disparaître dans un futur proche (Michel, 1998 : 218-223)?

Ne s'agirait-il pas d'abord d'un problème de discrimination envers les femmes et plus généralement envers les plus « faibles », auquel s'ajoute bien entendu une lutte pour le pouvoir absolu teintée d'ultra-capitalisme le plus sauvage, si souvent repérable et caractéristique dans l'univers masculin ? Citons l'exemple de Lamphan, jeune Thaillandaise de quinze ans originaire de la

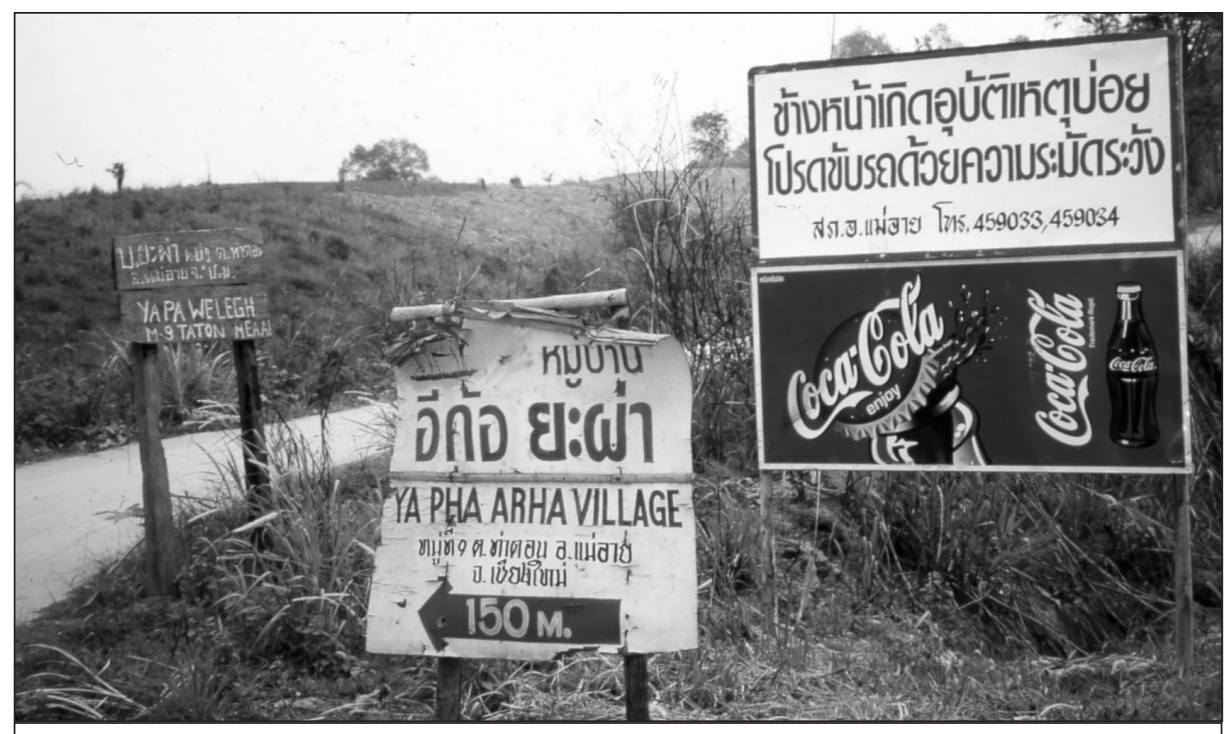

Tourisme ethnique et consommation : Entrée du village «traditionnel » de Ya La, Thaïlande. Photo: Franck Michel. 


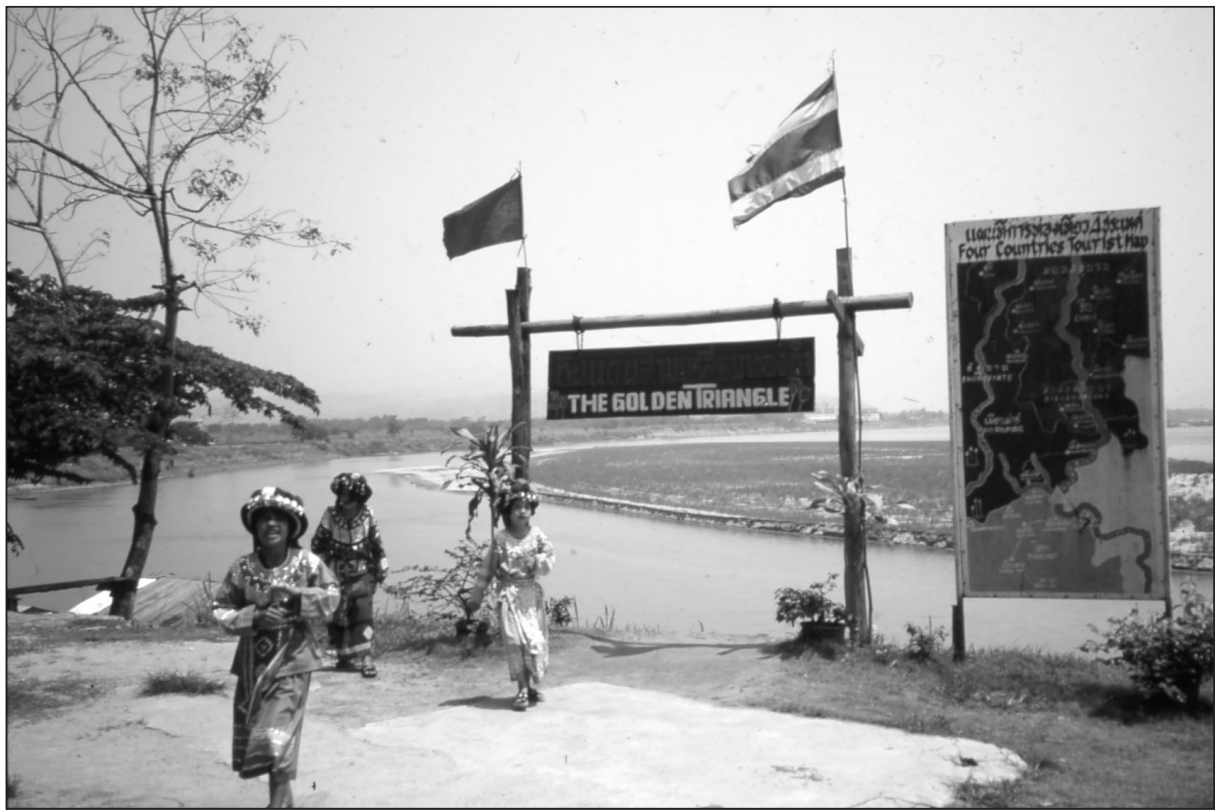

Jeunes filles Lisu déguisées au service de l'industrie touristique, Sop Ruak, Thaillande. Photo: Franck Michel.

province du Nord-Est, vendue par sa grandmère à un tenancier de bordel de Bangkok. Séquestrée, battue, Lamphan ne voulait qu'une seule chose : mourir. Elle a essayé de se suicider, mais ses geôliers ont réussi à l'en empêcher et le patron du bordel de lui répéter : "Je t'ai achetée et je peux faire tout ce que je veux avec toi $»$. Lamphan précise qu'au-delà de l'esclavage sexuel il y a d'abord le souci de nier l'humanité :

They tried to break my spirit. They said they wanted to teach me that I had to do whatever they wanted, at once, without thinking. So they starved me. They would lock me up for a couple of days and give me no food, only water, and they said they would give me food only when I agreed to give up. Then they bought out a plate of shit. Dog shit. They told me that I had to eat it. I wouldn't, and so they said that they would force me to eat it. They wanted to show that it was useless to fight back (cité dans Kristof, WuDunn, 2000 : 141).

Le proxénète est d'abord un «bon » capitaliste. Ne plus résister, capituler, se résigner, voilà ce qu'on exige en priorité de celle ou de celui qu' on opprime, alors que c'est tout ce qui reste à celles et à ceux qui n'ont plus rien... Seule éclaircie au tableau : Lamphan s'est échappée (après avoir été « re-livrée » par la police au patron du bordel une première fois...) et n'a pas contracté le virus du sida, une aubaine, si l'on compare à toutes les autres qui n'ont pas eu cette « chance »... Elle retournera pourtant à Bangkok, pour travailler dans une usine, car - une fois de plus - il n'y a pas d'argent au village...

Jeremy Seabrook (2001) dénonce avec force et raison les concomitances évidentes entre l'industrie du sexe et l'ensemble du secteur économique (auquel participe fortement le tourisme), ses liens mortifères à la source d'une forme d'ultra-capitalisme si vénérée en Asie, malgré la débâcle de l'été 1997 aux conséquences et aux raisons encore inavouables.

Économie en plein essor, l'industrie du sexe s'enrichit sur la misère du monde et représente en Thaillande la plus importante économie souterraine : le revenu annuel de ce secteur est évalué, en 1998, de 22,5 à 27 milliards de dollars américains, soit 10 à $14 \%$ du PNB (Courrier du Vietnam, 1998 : $6)$. Une forte hausse en moins de deux ans ! Heather Montgomery relève lors d'une enquête réalisée auprès des enfants prosti- tués en Thailande que «cette exploitation n'est pas advenue par la prostitution mais du fait de la pauvreté générale et de l'exclusion sociale » (1998 : 149). L'État s'est montré parfaitement incapable de s'occuper de « ses » enfants, explique l'auteur, des enfants-victimes qu'il faudrait savoir écouter avant de toujours vouloir parler à leur place, y compris dans le domaine sensible de la lutte contre la prostitution enfantine. En ce sens, Montgomery rejoint le chercheur thailandais Ing qui, dix ans avant lui, accusait sans détours l'État thailandais de proxénétisme, compte tenu notamment du pouvoir absolu de la police, de la corruption généralisée et des liens intrinsèques entre les forces de l'ordre et le milieu mafieux de la prostitution (1990 : 160). Dans ce domaine également, peu de choses semblent avoir changé depuis une décennie...

Le quotidien Libération du 27 novembre 1998 rapporte une étude du Bureau international du travail (BIT) qui considère qu' « en Thailande les femmes qui se prostituent dans les villes rapatrient près de 300 millions de dollars, par an, dans les zones rurales : un montant souvent supérieur aux budgets de développement financés par le gouvernement ». Un dit « miracle » économique cependant éphémère, dont la fragilité a éclaté au grand jour à compter de juillet 1997. Les chercheurs R. Bishop et L. Robinson insistent lourdement sur les responsabilités d'un ultra-libéralisme destructeur et arrogant ; ils évoquent le fossé croissant entre les ruraux et les citadins et les hordes de laissés-pourcompte qui hypothèquent fortement le « sauvetage » de l'économie nationale :

Ceci signifie plus de familles désespérées, davantage de femmes et plus de jeunes filles recrutées dans la prostitution, et des conditions de travail toujours pires (plus grande compétition, salaires moindres, menaces plus sérieuses sur l'état de santé, puisque le safe sex devient un luxe que bien peu de filles peuvent se permettre) (1998: 252).

Au-delà de la seule Thailande et même au-delà du tourisme de la dérive, c'est aussi de la prostitution en elle-même qu'il s'agit de débattre 
- sans passion excessive et sans concession arrangeante -, même si la discussion s'annonce sur ce thème évidemment moins consensuelle que celle qui a trait au seul tourisme sexuel concernant des enfants.

Nul doute, enfin, que pour juguler cette extension de la misère, on ne pourra faire l'économie de s'attacher aux vrais problèmes de la population locale : lutte contre la paupérisation criante, accès aux droits politiques et syndicaux, meilleure éducation et scolarisation, mise en garde contre le culte de l'argent qui autorise tous les dérapages, mobilisation contre l'exploitation éhontée des femmes et des enfants « utilisés » comme de simples et pratiques biens de consommation, etc. Le monde n'est pourtant pas une marchandise et le tourisme sexuel n'est pas une fatalité...

\section{Lutte contre la prostitution et prévention du sida}

Il conviendrait aujourd'hui, comme hier, d'éduquer, de rééduquer et encore d'éduquer plus et mieux toutes les «parties » concernées dans cette situation de crise : touristes, développeurs, voyagistes, prostituées, dirigeants, autochtones, etc., une très lourde tâche, pourtant primordiale, si l'on désire conjurer de tristes destins à venir et tenter de contenir l'épidémie de sida qui, en Thailande, a officiellement tué 66000 personnes en 1999. Cette même année, on recense dans tout le royaume 755000 personnes porteuses du virus HIV (dont 13900 enfants de 0 à 15 ans). Le Nord du pays apparaît - proportionnellement à sa population - indiscutablement comme le plus affecté. Des chiffres sans doute encore à revoir à la hausse ${ }^{2} .$.

Pourtant, d'autres batailles autour de la législation portent des fruits. Et les choses commencent timidement à bouger même si cela ne correspond, pour l'instant, qu'à une goutte d'eau dans un océan de misère et de collusion... Prenons le cas de la lutte contre la pédophilie et des réelles avancées judiciaires, notamment en matière de poursuites dans le pays de l'accusé à propos de crimes commis à l'étranger (Seabrook, 2000). Ainsi, pour ne prendre l'exemple que de la France, la condamnation le 20 oc- tobre 2000 d'Amnon Chemouil à sept ans de prison (pour le viol d'une fillette de onze ans sur le sol thaïlandais) constitue une avancée indéniable, quoiqu'un peu symbolique, dans la lutte contre le tourisme sexuel. Rappelons que, lors du procès Chemouil, $\mathrm{M}^{\mathrm{e}}$ William Bourdon, conseil de l'Association contre la prostitution enfantine, avait dit : "Cet homme ne peut et ne doit pas payer pour tous ceux qui n'ont pas payé » ; mais ensuite toutes les parties civiles représentées s'étaient entendues pour affirmer qu' " un visa pour la Thaïlande n'est pas un visa pour l'impunité »(Le Monde, 22-23 octobre 2000). En France, l'évolution de l'arsenal juridique (avec la loi du 17 juin 1998, plus répressif en matière d'infraction de nature sexuelle) contribue à ce combat et Chemouil a été le premier à « inaugurer » l'application de cette nouvelle loi. Pourtant, le constat reste amer : en France seulement, combien sont-ils passés entre les mailles du filet de la justice, de juin 1998 à aujourd'hui ? 50, 500, 5000 ou 50000 ? Funeste pronostic bien difficile à établir. La lutte contre cette forme de tourisme abject doit toutefois passer par une meilleure éducation des voyageurs, une éthique plus responsable de la part des voyagistes, des campagnes de sensibilisation efficaces, plutôt que des verdicts spectaculaires ou exemplaires. L'enseignement fondamental qu'il nous incombe de méditer plus avant, si nous désirons efficacement combattre le fléau du tourisme sexuel à destination des enfants, réside dans la prise de conscience globale stipulant que la plupart des gens qui exercent des abus sexuels sur des enfants ne sont pas des pédophiles, mais des personnes « ordinaires ».

\section{Conclusion}

Les principaux analystes de la situation sociale en Thailande sont unanimes à considérer que l'avenir paraît pour le moins morose, comme le constatent aussi Bishop et Robinson dans leur étude sur les interactions entre culture sexuelle et miracle économique :

Si le tourisme doit effectivement être aussi central pour la renaissance économique thaïlandaise qu'il l'a été pour l'ère du développement rapide - et il peut difficilement en aller autrement puisque les projets en cours ne

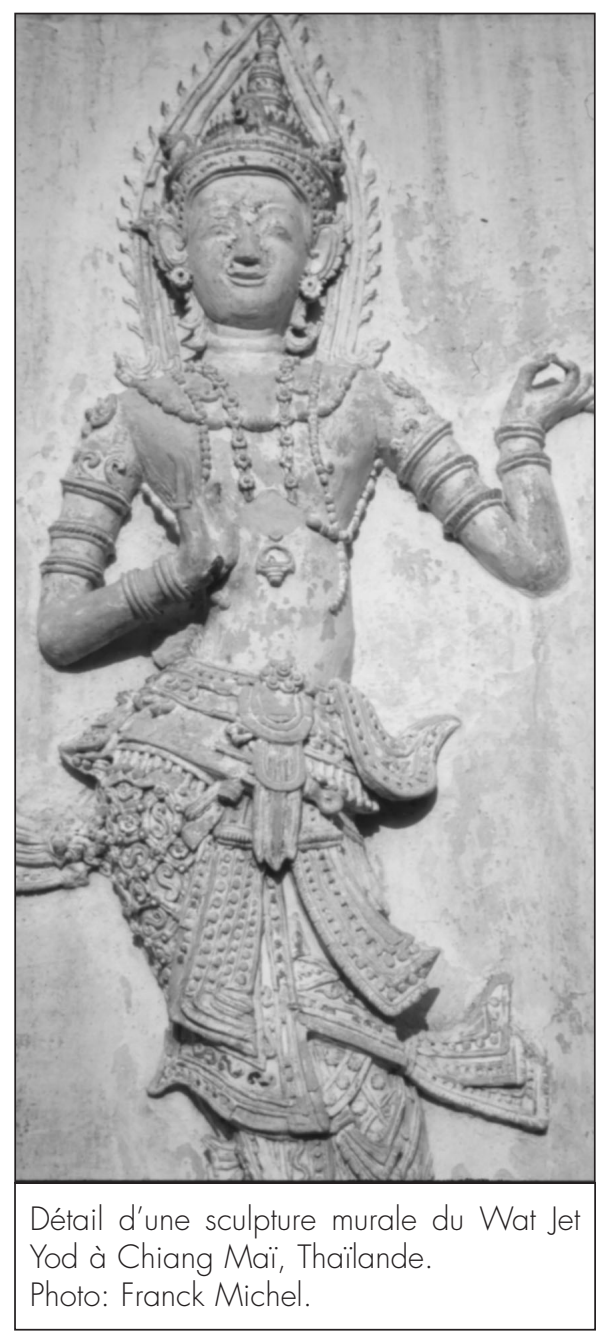

conçoivent pas de nouvelle orientation en matière de politique industrielle ou agricole -, le sexe continuera à être essentiel pour le tourisme ainsi que pour le redressement économique de la nation (1998: 251).

Les auteurs analysent ce trop fameux « miracle » thaïlandais, un temps modèle des capitalistes les plus fervents, qui s'est avéré en fait reposer sur l'exploitation à outrance des femmes, des enfants, des minorités, des plus démunis, le tout sur fond de corruption, endémique et généralisée.

Constatant la banalisation du commerce du sexe en Thailande, Thanh-Dam Truong écrivait dès la fin des années 1980 : «Cela reflète aussi l'échec des politiques de développement visant à améliorer le niveau de vie des pauvres en milieu rural et urbain, et à réduire le taux d'exploitation sur le mar- 
ché du travail, particulièrement en ce qui concerne le respect du travail féminin »; il précisait qu'au royaume de Siam « une très jeune fille peut être 'louée' pour trois heures à 85 'guichets' pour 35 \$US» (1989: 342-343). La situation ne s'améliorera guère au cours des deux décennies suivantes ; elle s'adaptera plutôt aux nouvelles réalités du marché et au contexte géopolitique reconfiguré... Ainsi, au milieu des années 1990, nous avons souligné l'aggravation - et les conséquences dramatiques - de la prostitution à des fins touristiques (Michel, 1995 : 182-201), situation qui, avec la crise économique de 1997 et les turpitudes du consumérisme et de la mondialisation, s'est encore détériorée ces toutes dernières années, bien en dépit d'une réelle amélioration des législations en vigueur (mais les nouvelles lois sur la prostitution ou sur la lutte contre la pédophilie et le trafic d'enfants ne sont que rarement appliquées) et d'une prise de conscience évidente de la part de la population thailandaise, notamment en raison des ravages du sida... Mais, selon d'autres chercheurs, dont Bello, la situation est critique et la Thaïlande sera prochainement - triste record - le seul pays du globe dont la population devrait diminuer en raison du sida (1998 : 224). La prostitution touristique en Thailande est-elle donc un syndrome tant singulier qu' extrême de la misère noire d'une certaine mondialisation? Sans doute...

Franck Michel est anthropologue et directeur de la revue Histoire \& Anthropologie (Strasbourg), co-responsable du Centre de Recherche sur le Voyage (Strasbourg) et associé au bureau de chercheurs et d'auteurs Homnisphères (Paris). Son plus récent ouvrage est Désirs d'Ailleurs. Essai d'anthropologie des voyages, Éd. Histoire \& Anthropologie, 2002

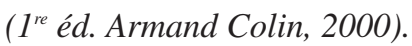

\section{Notes}

1 Soldat de l'armée américaine.

2 Source : site Internet unaids.org, janvier 2001. Ces données nous apprennent également que le taux d'infection s'élève en Thaïlande à un peu plus de $2 \%$ de la population adulte. Parmi les Commercial Sex
Workers, ceux de Bangkok semblent être mieux protégés que par le passé ; par contre, les prostitués hommes et femmes du Nord de la Thailande sont nettement les plus exposés. De même en est-il de leurs clients. Voici ce que dit à ce sujet le rapport officiel des Nations Unies : "In Bangkok, HIV prevalence among sex workers tested (both direct and indirect combined) has ranged from 7 to 18 percent between 1990 and 1996. Since 1994, HIV prevalence in this group has declined slightly from 13 to 7 percent in 1997. Outside of Bangkok, HIV prevalence among sex workers tested (both direct and indirect) increased from 5 percent to 20 percent. HIV prevalence rates are highest in the northern provinces ranging from 16 to 57 percent among direct sex workers. [...] Outside of Bangkok, HIV prevalence among male STD clinic patients tested increased from 3 to 9 percent between 1990 and 1996. In 1997, the median rate decreased to 7 percent. Again, there is a significant north-south gradient with HIV prevalence over 40 percent among male STD clinic patients in the northern most provinces ».

\section{Bibliographie}

"L'industrie du sexe : une économie en plein essor », Le Courrier du Vietnam, Hanoï, 30 août 1998, p. 6.

Bello, W. (dir.) (1998), A Siamese Tragedy. Development and Disintegration in Modern Thailand, Londres, Zed Books.

Boonchalaksi, W., et P. Guest (1994), Prostitution in Thailand, Bangkok, Mahidol University.

Brown, L. (2000), Sex Slaves. The Trafficking of Women in Asia, Londres, Virago Press.

Bishop, R., L.S. Robinson (1998), Night Market. Sexual Cultures and the Thai Economic Miracle, Londres, Routledge.

Cazes, G. (1983), Le tourisme international en Thaïlande et en Tunisie. Les impacts et les risques d'un développement mal maîtrisé, Reims, Travaux de l'Institut de géographie de Reims, doc. 53-54.

Formoso, B. (2001), « Corps étrangers. Tourisme et prostitution en Thaïlande », Anthropologie et Sociétés, Québec, Université Laval, Vol. 25, $\mathrm{n}^{\circ}$ 2, $4^{\mathrm{e}}$ trimestre, p. 55-70.

Formoso, B. (2000), Thaïlande. Bouddhisme renonçant, capitalisme triomphant, Paris, La Documentation française.

Ing, K. (1990), « Une honte mal placée », dans G. Franco (dir.), Thaïlande. Les larmes $d u$ Bouddha, Paris, Autrement, n 43, p. 161-164.
Kristof, N.D., et S. WuDunn (2000), Thunder from the East. Portrait of a Rising Asia, Londres, Nicholas Brealey Publishing.

Michel, F. (2002 [2000]). Désirs d'Ailleurs. Essai d'anthropologie des voyages, Strasbourg, Éd. Histoire \& Anthropologie.

Michel, F. (1995), En route pour l'Asie. Le rêve oriental chez les colonisateurs, les aventuriers et les touristes occidentaux, Strasbourg, Éd. Histoire \& Anthropologie.

Michel, F. (1998), « Le tourisme sexuel en Asie : du cauchemar à l'horreur », dans F. Michel (dir.), Tourismes, touristes, sociétés, Paris, L'Harmattan, p. 207-234.

Montgomery, H. (1998), « Children, Prostitution and Identity. A Case Study from a Tourist Resort in Thailand ", dans K. Kempadoo et J. Doezema (dir.), Global Sex Workers, Londres, Routledge, p. 139-150.

Phongpaichit, P. (1998), Guns, Girls, Gambling, Ganja, Thailand's Illegal Economy and Public Policy, Chiang Mai, Silkworms Books.

Poona, Antaseeda (1997), « More Foreigner Workers Join Sex Industry », Bangkok Post, 27 novembre, p. 4.

Sanitsuda, E. (1993 [1990]), Behind the Smile. Voices of Thailand, Bangkok, The Post Publishing Co.

Seabrook, J. (2001 [1996]), Travels in the Skin Trade. Tourism and the Sex Industry, Londres, Pluto Press.

Seabrook, J. (2000), No Hiding Place. Child Sex Tourism and the Role of Extraterritorial Legislation, Londres, Zed Books.

Truong, T.D. (1990), Sex, Money and Morality. Prostitution and Tourism in Southeast Asia, Londres, Zed Books.

Truong, T.D. (1989), « The Dynamics of Sex Tourism: The Case of Southeast Asia », dans T. V. Singh, H. L. Theuns et F.M. Go (dir.), Towards Appropriate Tourism: The Case of Developing Countries, Frankfort, Peter Lang. 